\title{
Tópicos Emergentes em Insuficiência Cardíaca: O Futuro na Insuficiência Cardíaca: Telemonitoramento, Wearables, Inteligência Artificial e Ensino na Era Pós-Pandemia
}

\author{
Emerging Topics in Heart Failure: The Future of Heart Failure: Telemonitoring, Wearables, Artificial Intelligence \\ and Learning in the Post-Pandemic Era
}

Aguinaldo F. Freitas Jr., ${ }^{\oplus}$ Fábio S. Silveira, ${ }^{2,3}$ Germano E. Conceição-Souza, ${ }^{4,5}{ }^{\oplus}$ Manoel F. Canesin, ${ }^{6,7}$ Pedro V. Schwartzmann, ${ }^{8,9}$ Sabrina Bernardez-Pereira, ${ }^{10}$ Reinaldo B. Bestetti11@

Hospital das Clínicas da Universidade Federal de Goiás (HC-UFG), ${ }^{1}$ Goiânia, GO - Brasil

Fundação Beneficência Hospital de Cirurgia (FBHC-Ebserh), ${ }^{2}$ Aracaju, SE - Brasil

Centro de Pesquisa Clínica do Coração, ${ }^{3}$ Aracaju, SE - Brasil

Hospital Alemão Oswaldo Cruz, ${ }^{4}$ São Paulo, SP - Brasil

Hospital Regional de São José dos Campos, ${ }^{5}$ São José dos Campos, SP - Brasil

Hospital Universitário - Universidade Estadual de Londrina (HU-UEL), ${ }^{6}$ Londrina, PR - Brasil

ACTIVE - Metodologias Ativas de Ensino, ${ }^{7}$ São Paulo, SP - Brasil

Hospital Unimed Ribeirão Preto, ${ }^{8}$ Ribeirão Preto, SP - Brasil

Centro Avançado de Pesquisa, Ensino e Diagnóstico (Caped), ${ }^{9}$ Ribeirão Preto, SP - Brasil

Hospital de Coração (HCor), ${ }^{10}$ São Paulo, SP - Brasil

Departamento de Medicina, Universidade de Ribeirão Preto (Unaerp), ${ }^{11}$ Ribeirão Preto, SP - Brasil

Carta científica referente ao Heart Failure Summit Brazil 2020 / Departamento de Insuficiência Cardíaca - DEIC/SBC

Telemonitoramento na Insuficiência Cardíaca (IC) e Manejo Remoto

O telemonitoramento consiste no monitoramento e suporte à distância (TMO) do paciente com IC crônica. O TMO pode ser não invasivo (TMONI) ou invasivo (TMOI). O TMONI compreende ligações telefônicas, orientações periódicas por meio de material instrutivo, controle e monitoramento do peso corporal, vídeo - chamadas e teleconsultas. ${ }^{1,2}$ O TMOI envolve uma série de dispositivos implantáveis que transmitem, a um servidor à distância, informações hemodinâmicas e impedância intratorácica. ${ }^{3,4}$

Ensaios clínicos de TMONI costumam ter resultados conflitantes. No entanto, metanálises envolvendo estudos observacionais e randomizados de TMOI e TMONI têm mostrado impacto positivo no prognóstico de pacientes com IC. A redução na mortalidade geral pode variar de 19 a 31\% com o TMOI ou TMIONI em pacientes com IC, ${ }^{5,6}$ enquanto a redução na frequência de internação hospitalar por IC varia de 27 a 39\%, principalmente em pacientes em classe funcional III/IV. ${ }^{7-9}$

\section{Palavras-chave}

Telemonitoramento, Inteligência Artificial, Insuficiência Cardíaca, Ensino.

Correspondência: Reinaldo B Bestetti •

Universidade de Ribeirão Preto, Curso de Medicina - Av. Costabile Romano, 2201. CEP 14096-900, Ribeirão Preto, SP - Brasil

E-mail rbestetti44@gmail.com

Artigo recebido em 20/10/2020, revisado em 20/10/2020, aceito em 20/10/2020

DOI: https://doi.org/10.36660/abc.20201127
O TMOI com evidências mais convincentes na IC é o CardioMEMS, ${ }^{7}$ um dispositivo implantado via percutânea na artéria pulmonar, que transmite os valores pressóricos centrais para um servidor seguro e orienta o ajuste nas doses de diuréticos e vasodilatadores.

\section{Wearables na IC: Ferramentas de Monitoramento ou Gadget Eletrônico?}

Wearables são ferramentas computacionais "vestíveis". Podem ser um relógio, uma camisa, uma lente de contato ou um sapato, por exemplo. Esses aparelhos contêm sensores que podem obter dados em tempo real e transmiti-los para uma nuvem ou para outro dispositivo, permitindo análises de uma enorme quantidade de dados, bem como a sua disponibilização para decisões diagnósticas e terapêuticas. Tudo isso é permitido graças à evolução da tecnologia de transmissão de dados, com o advento do 5G.

Assim, a Internet das coisas (IoT) realmente se tornará progressivamente uma realidade em diversos países. Com a Internet das coisas médicas (IoMT), não será diferente. Com o progressivo barateamento dessas tecnologias, vencendose a barreira do custo-efetividade, teremos a oportunidade de testar uma infinidade de wearables que poderão permitir o acesso precoce da equipe de saúde a dados de telemonitoramento de variáveis como pressão arterial, pulso, saturação de oxigênio, análise postural, queda, frequência respiratória, temperatura, glicemia capilar, entre outros.

Isso poderá impactar em desfechos clinicamente relevantes, como hospitalizações, custos diretos e indiretos e até mortalidade, ao mesmo tempo em que se poderá direcionar o manejo dos pacientes com IC para uma medicina de precisão mais personalizada - um novo paradigma. Indubitavelmente, há a necessidade de validação de cada 
gadget que se proponha a trazer estes benefícios, levando em consideração as principais barreiras para sua implementação, mas definitivamente os wearables chegaram para ficar. ${ }^{8,9}$

\section{Inteligência Artificial e Big Data na IC}

Sistemas computacionais capazes de realizar tarefas que originalmente exigiam atuação humana constituem a base da inteligência artificial (IA). Esses sistemas surgiram pela necessidade de interpretação de grande volume de dados (big data). Os sistemas devem ser capazes de analisar dados simples ou complexos, de forma veloz e com acurácia, além de se adaptarem a eles, sem uma programação estática. ${ }^{10}$ Machine learning (ML) e deep learning (DL) são extensões da IA. O ML é a prática de usar algoritmos para coletar dados, aprender com eles e então fazer a determinação ou predição de alguma coisa, de pessoas ou mesmo de pacientes. Para ser útil e fidedigno, precisa ser constantemente alimentado por dados reais. Além disso, o DL, que é o extremo da interação e adaptação de aprendizado, pode estabelecer conexões neurais e diversidade para integrar diferentes bases de dados. ${ }^{11}$

A IA, a ML e o DL já apresentam aplicações em estudo para IC, seja como diagnóstico, avaliação de prognóstico, TMO ou ainda para selecionar pacientes com maior benefício para diversas terapias. Isso pode ser feito, por exemplo, na distinção de fenótipos, alocando pacientes em diferentes perfis de assinatura de doença; ${ }^{12}$ na melhor acurácia para o diagnóstico de IC aguda em relação ao médico; ${ }^{13}$ e no eventual direcionamento para terapias novas ou já estabelecidas, como análise adicional do ECG basal para identificar paciente melhor respondedor à TRC. ${ }^{14}$

\section{Ensino Médico em IC nos Novos Tempos}

Um dos grandes desafios dos estudos e pesquisa clínica é a sua capacidade de traduzi seus resultados científicos para a prática clínica. São vários os fatores envolvidos nesse processo; um importante fator é, particularmente, a capacidade de transmissão e aplicação efetiva em menor intervalo de tempo desse conhecimento para um maior número de profissionais.

As mudanças em ensino médico e, consequentemente, no tema IC, passam por uma revolução. ${ }^{15} \mathrm{O}$ que há pouco tempo se resumia quase que exclusivamente a aulas expositivas, há anos, através de técnicas de metodologia ativa de ensino e mais recentemente por modelos híbridos sincrônicos ou assíncronos digitais, o ensino é aperfeiçoado, aprimorando o processo de aprendizagem e, consequentemente, o cuidado com o paciente. ${ }^{16}$

O modelo atual de ensino deve oferecer o conceito de andragogia, ${ }^{17}$ associado ao modelo de ensino AGES. Esse modelo engloba a construção de processos expositivos de aprendizagem curtos, que mantêm a atenção do expectador ou de alunos, associado à capacidade de geração de motivos intrínsecos que tragam significado na aprendizagem. Ou seja, envolve emoções que possam fortalecer o aprendizado, de forma que este seja construído em várias etapas (Spaced). Quando incorporados, todos estes pontos formam uma aprendizagem mais profunda e efetiva, levando ao médico e ao profissional de saúde a possibilidade de adquirir e aplicar na prática os cuidados em IC. ${ }^{18}$

Lista de Participantes do Heart Failure Summit Brazil 2020 / Departamento de Insuficiência Cardíaca - DEIC/SBC

Aguinaldo Freitas Junior, Andréia Biolo, Antonio Carlos Pereira Barretto, Antônio Lagoeiro Jorge, Bruno Biselli, Carlos Eduardo Montenegro, Denilson Campos de Albuquerque, Dirceu Rodrigues de Almeida, Edimar Alcides Bocchi, Edval Gomes dos Santos Júnior, Estêvão Lanna Figueiredo, Evandro Tinoco Mesquita, Fabiana G. Marcondes-Braga, Fábio Fernandes, Fabio Serra Silveira, Felix José Alvarez Ramires, Fernando Atik, Fernando Bacal, Flávio de Souza Brito, Germano Emilio Conceição Souza, Gustavo Calado de Aguiar Ribeiro, Humberto Villacorta Jr., Jefferson Luis Vieira, João David de Souza Neto, João Manoel Rossi Neto, José Albuquerque de Figueiredo Neto, Lídia Ana Zytynski Moura, Livia Adams Goldraich, Luís Beck-da- Silva, Luís Eduardo Paim Rohde, Luiz Claudio Danzmann, Manoel Fernandes Canesin, Marcelo Bittencourt, Marcelo Westerlund Montera, Marcely Gimenes Bonatto, Marcus Vinicius Simões, Maria da Consolação Vieira Moreira, Miguel Morita Fernandes da Silva, Monica Samuel Avila, Mucio Tavares de Oliveira Junior, Nadine Clausell, Odilson Marcos Silvestre, Otavio Rizzi Coelho Filho, Pedro Vellosa Schwartzmann, Reinaldo Bulgarelli Bestetti, Ricardo Mourilhe Rocha, Sabrina Bernadez Pereira, Salvador Rassi, Sandrigo Mangini, Silvia Marinho Martins, Silvia Moreira Ayub Ferreira, Victor Sarli Issa.

\section{Contribuição dos Autores}

Redação do manuscrito: Freitas Jr. AF, Silveira FS, Conceição-Souza GE, Canesin MF, Schwartzmann PV, Bernardez-Pereira S, Bestetti RB; Revisão crítica do manuscrito quanto ao conteúdo intelectual importante: Bernardez-Pereira S, Bestetti RB.

\section{Potencial Conflito de Interesses}

Declaro não haver conflito de interesses pertinentes.

\section{Fontes de Financiamento}

O presente estudo não teve fontes de financiamento externas.

\section{Vinculação Acadêmica}

Não há vinculação deste estudo a programas de pósgraduação. 


\section{Referências}

1. Mesquita ET, Queluci GC. Abordagem multidisciplinar ao paciente com insuficiência cardíaca. São Paulo: Atheneu; 2013.

2. Lopes MAC, Oliveira GMM, Ribeiro ALP, Pinto FJ, Rey HCV, Zimerman LI, et al. Diretriz da Sociedade Brasileira de Cardiologia sobre Telemedicina na Cardiologia - 2019. Arq Bras Cardiol. 2019; 113(5):1006-1056.

3. Hindricks G, TaborskyM, Glikson M, et al. Implant-based multiparameter telemonitoring of patients with heart failure (IN-TIME): a randomised controlled trial. Lancet. 2014;384:583-90.

4. KurekA, TajstraM, Gadula-Gacek E, et al. Impact of remote monitoring on long-term prognosis in heart failure patients in a real-world cohort: results from all-comers COMMIT-HF trial. J Cardiovasc Electrophysiol. $2017 ; 28: 425-31$.

5. Lin MH, Yuan WL, Huang TC, Zhang HF, Mai JT, Wang JF. Clinical effectiveness of telemedicine for chronic heart failure: a systematic review and metanalysis. J Invest Med 2017; 65:899-911.

6. Koehler F, Koehler K, Deckwart O, Prescher S, Wegscheider K, Kirwan BA, et al. Efficacy of telemedical intervention management in patients with heart failure (TIM-HF2): a randomised, controlled, parallel-group, unmask trial. Lancet 2018; 392: 1047-1057.

7. Abraham WT, Stevenson LW, Bourge RC, et al. Sustained efficacy of pulmonary artery pressure to guide adjustment of chronic heart failure therapy: complete follow up results from the $\mathrm{CHAMPION}$ randomised trial. Lancet. 2016;387:453-61

8. Sighal A, Cowie MR. The role of wearables in heart failure. Curr Heart Fail Rep. 2020(4):125-132.

9. DeVore AD, Wosik J, Hernandez AF. The future of Wearables in Heart Failure Patients. JACC Hear Fail. 2019(11):922-932.
10. Awan SE, Sohel F, Sanfilippo FM, Bennamoun M, Dwivedi G. Machine learning in heart failure: ready for prime time. Curr Opin Cardiol. 2018;33(2):190-5.

11. de Marvao A, Dawes TJ, Howard JP, O'Regan DP. Artificial intelligence and the cardiologist: what you need to know for 2020 . Heart. 2020;106(5):399-400.

12. Ahmad T, Wilson FP, Desai NR. The Trifecta of Precision Care in Heart Failure: Biology, Biomarkers, and Big Data. J Am Coll Cardiol. 2018;72(10):1091-4.

13. Choi DJ, Park JJ, Ali T, Lee S. Artificial intelligence for the diagnosis of heart failure. NPJ Digit Med. 2020;3:54.

14. Feeny AK, Rickard J, Trulock KM, Patel D, Toro S, Moennich LA, et al. Machine Learning of 12-Lead QRS Waveforms to Identify Cardiac Resynchronization Therapy Patients With Differential Outcomes. Circ Arrhythm Electrophysiol. 2020;13(7):e008210.

15. Canesin MF, Oliveira Jr MT, Barretto ACP, Nazima WI, Gualandro DM, C. Magalhaes C, Ferreira JF, D. Ferreira, Cardoso JCS: European Heart Journal (2014) 35 (Abstract Supplement), 836.

16. Flodgren $\mathrm{G}, \mathrm{O}^{\prime}$ Brien MA, Parmelli E, Grimshaw JM. Local opinion leaders: effects on professional practice and healthcare outcomes. Cochrane Database of Systematic Reviews 2019, Issue 6. Art. No.: CD000125. DOI: 10.1002/14651858.CD000125.pub5

17. The Adult Learner: The Definitive Classic in Adult Education and Human Resource Development Mar 23, 2011; Malcolm S. Knowles, Richard A. Swanson, Elwood F. Holton III Ed.D.

18. Anderson, L.W. (Ed.), Krathwohl, D.R. (Ed.), Airasian, P.W., Cruikshank, K.A., Mayer, R.E., Pintrich, P.R., Raths, J., \& Wittrock, M.C.: 2001, A taxonomy for learning, teaching, and assessing: A revision of Bloom's Taxonomy of Educational Objectives (Complete edition). New York: Longman. 